\title{
Liquid Biopsy: a new tool to overcome CDKi resistance mecha- nism in Luminal metastatic breast cancer
}

\author{
Miriam González-Conde ${ }^{1}$, Celso Yañez ${ }^{1}$, Rafael López-López ${ }^{1,2,3}$ and Clotilde Costa1,3,*
}

1 Roche-Chus Joint Unit, Translational Medical Oncology Group, Oncomet, Health Research Institute of Santiago de Compostela (IDIS), Travesía da Choupana s/n, 15706 Santiago de Compostela, Spain.

Clotilde.costa.nogueira@sergas.es, Miriam.gonzalez.conde@sergas.es, Celso.yanez.gomez@sergas.es

2. Medical Oncology Department, University Clinical Hospital of Santiago de Compostela, 15706 Santiago de Compostela, Spain. Translational Medical Oncology Group (Oncomet), Health Research Institute of Santiago de Compostela (IDIS), 15706 Santiago de Compostela, Spain. Rafael.lopez.lopez@sergas.es

3. CIBERONC, Centro de Investigación Biomédica en Red Cáncer, 28029 Madrid, Spain.

*Correspondence: Clotilde.costa.nogueira@sergas.es; Tel.: +34-981-955-602

Abstract: Breast cancer is the most commonly diagnosed cancer in women worldwide. Approximately, $70 \%$ of breast cancer patients express hormone receptors (HR) (Luminal subtype). Adjuvant endocrine treatments are the standard of care in HR+/HER2- breast cancer. Over time, about $50 \%$ of those patients develop endocrine resistance and metastatic breast cancer. Cyclin-dependent kinase inhibitors (CDKi) in combination with an aromatase inhibitor or fulvestrant have demonstrated superior efficacy increasing progression-free survival, with a safe toxicity profile, in HR+/HER2- metastatic breast cancer patients. CDKi blocks kinases 4/6 ATP-binding domain preventing G1/S cell cycle transition. Despite this, not all patients respond to CDKi and those who respond, finally develop resistance to combination therapy. Different studies, in tumour tissue or cell lines, have tried to elucidate the mechanisms underlying this progression, but there are still no conclusive data. In the last few years, liquid biopsy has contributed relevant information to this knowledge. Liquid biopsy can be performed in real-time, non-invasively and be repeated whenever needed. Circulating tumour material are potential prognostic markers in metastatic luminal breast cancer to determine patient prognosis, monitor disease and treatment selection. The objective of this review is to outline the different studies carried out in HR+ metastatic breast cancer patients treated with CDKi plus endocrine therapy using liquid biopsy approaches looking for possible resistance mechanisms.

Keywords: breast cancer, CDK inhibitors, liquid biopsy, resistance mechanisms, therapy 


\section{Introduction}

Breast cancer (BC) is the most common cancer and the second cause of cancer death among women worldwide, mainly to metastasis. The incidence is more than 2 million people with a 5 -year prevalence [1]. There are different breast cancer subtypes: hormone receptor (HR+) (luminal A and luminal B), human epidermal receptor 2 (HER2) enriched, and basal-like or triple-negative (TNBC), defined by the lack of estrogen (ER) and progesterone receptor (PR) and the absence of HER2 protein overexpression [2]. The Luminal subtype represents approximately $70 \%$ of the cases and it is characterized by the expression of hormone receptors; estrogen and progesterone [3]. Hormone receptor-positive $(\mathrm{HR}+)$ patients are treated with endocrine therapies (ET) such as tamoxifen, anastrozole, letrozole or exemestane, to block the hormone receptor or inhibit systemic estrogen production. Most of the patients diagnosed with primary luminal BC are treated by ET adjuvantly to surgery and or radiotherapy [4], but it was estimated that $30 \%$ of patients will develop metastasis, while $6 \%$ already have metastatic disease at the time of diagnosis [5], [6].

Different mechanisms of endocrine resistance have been identified such as upregulation of cyclins, cyclin-dependent kinases (CDKs) and mitogen signalling pathways (PI3K and RAS), reduction of CDK inhibitory proteins (p16, p21, p27), mutations or loss of ESR1 as well as epigenetic alterations [7]. CDKs act downstream of estrogen signalling, controlling cell cycle progression. As these proteins are normally altered in breast cancer, have been considered a key target for therapeutic intervention in the metastatic setting [6].

The Food and Drug Administration (FDA) and The European Medicines Agency (EMA) approved the combination of cyclin-dependent kinase inhibitors (CDKi) with endocrine therapy to treat advanced luminal BC. So far three different inhibited cyclins called palbociclib, ribociclib and abemaciclib have been marketed. Clinical trials [8-13] have demonstrated that the CDK4/6 inhibitors plus endocrine therapy (ET) combination improves progression free survival and survival rates compared with ET alone. Nevertheless, not all patients respond to CDKi and those who initially respond, ultimately progress. There are factors responsible for endocrine resistance that have not yet been identified [14], which complicates the study of resistance combining both therapies. In addition, preclinical studies suggest that the heterogeneous resistance to CDKi further hinder decipher resistance mechanisms for the combinatorial therapy [15]. The efforts made using mainly primary tumour tissue samples or cell lines did not get conclusive results. Therefore, a change in the paradigm is needed for this emergent drug-resistant patients group. Precision oncology throughout the analysis of liquid biopsies emerges as an attractive opportunity for it. Contrary to classical oncology, the therapeutic strategy on precision medicine is based on the distinctive molecular characteristics of patients. Thus, the objective is to tailor patient therapy studying biomarker profiles while reducing, as much as possible, the harmful effects on healthy cells. The latter was already done in BC, where there are a selective treatment depending on tumour subtype. Thus, luminal patients are treated with endocrine therapy while HER2 patients will receive anti-HER2 antibodies[16].

In last years, liquid biopsy is being studied as a tool to comprehend tumour evolution in real-time to guide systemic treatment selection for precision medicine. Moreover, it provides information on the genomic profile of a given cancer and an assessment of tumour burden, without the need for invasive procedures. Although the analytical and clinical validity of the liquid biopsy is increasingly evident, clinical trials that incorporate the analysis of tumour-derived material as ctDNA or CTCs are still necessary for clinic decision-making. Thus, the detection of PIK3CA mutations in plasma ctDNA to guide treatment with the PIK3CA inhibitor alpelisib could provide the first example of a clinically useful ctDNA assay in clinic. 
A review of published literature was conducted to assess the use of Liquid Biopsy analysing tumour-derived material (ctDNA, CTC and extracellular vesicles) to identify biomarkers that predict resistance in HR+/HER2- MBC patients treated with CDK 4/6 inhibitors plus endocrine therapy.

\section{Inhibition of the Cyclin-Dependent Kinases 4/6 (CDK4/6) in combination with endocrine therapy for HR+/HER2- metastatic Breast Cancer}

The cell cycle is divided into different phases: G1, S (DNA synthesis), G2 and M (mitosis). Transitions between these phases are controlled by cyclin-dependent kinases and cyclins. G1-S transition is controlled by CCND1-CDK4/6 complex [17], [18]. The CCND1-CDK4/6-RB1-INK4 axis is normally up-regulated in HR+/HER2- BC, being common an amplification in CCND1 (29 \% in Luminal A and B) and CDK4 (14\% Luminal A and $25 \%$ Luminal B). Thus, mitogenic and oncogenic signalling cascades activate different cyclins, mainly CCND1. CCND1-CDK4/6 complex phosphorylates the retinoblastoma protein $(\mathrm{RB})$, a negative regulator of cell cycle progression. Inactivation of $\mathrm{RB}$ releases E2F transcription factor, which activates the transcription of genes required for DNA replication and cell cycle progression [4], [17], [19], [20] (Figure 1).

Inhibition of CDK4/6 leads to G1 arrest. Pharmaceutical companies envisioned the opportunity and designed treatments targeting CDK4/6. The first generation of CDKi was non-specific and had limited efficacy, affinity and considerable toxicity[6], [17], [21]. Currently, computer-aided drug design is used to develop CDK inhibitors with better potency, selectivity and pharmacological properties studying the spatial structure and inhibition activity of CDKs [22], [23]. Palbociclib, ribociclib and abemaciclib are the last generations of CDK inhibitors that target the ATP binding domain of CDK4/6. The chemical structure determines the specificity against cyclin-dependent kinases, having palbociclib and ribociclib more than 100 fold-higher affinities with CDK4/6 while abemaciclib only 6 fold higher affinity. A more profound understanding of molecular differences is necessary for the precise use of this drugs in the clinic setting, although it was confirmed the comparable efficacy of these inhibitors by increasing Progression Free Survival (PFS) independently of patients features [24]. 


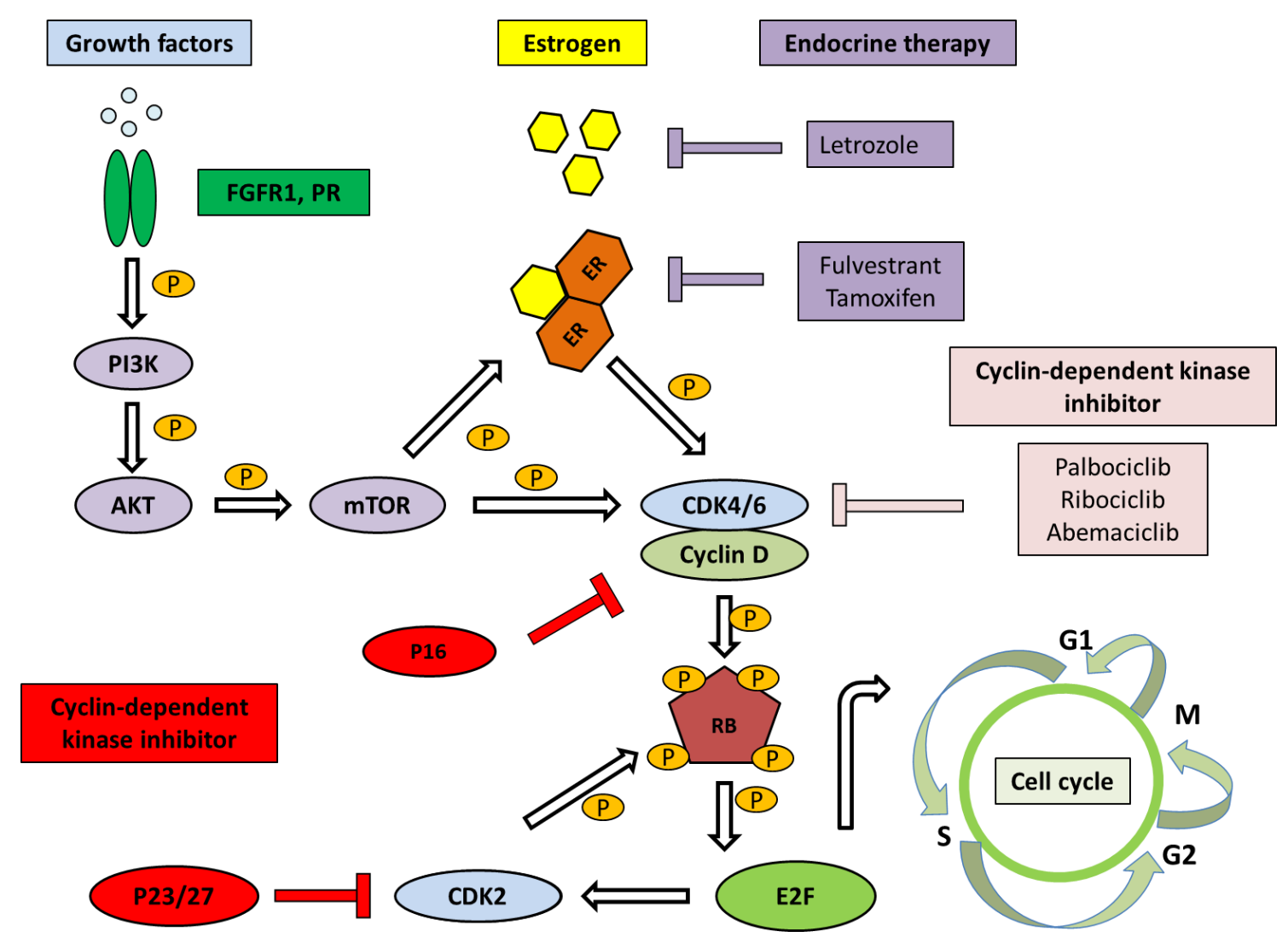

Figure 1. Representation of the regulation of the cell cycle and the different targets for CDKi and endocrine therapies.

MBC patients treated with CDKi who had previously been treated with two or more hormonal line treatments had a higher clinical benefit rate and PFS. It was also observed that the response to therapy was independent of $R B 1$ nuclear expression, the Ki-67 index, p16 loss or the amplification of CCND1 in the tumour tissue. Due to synergetic effect between $\mathrm{ET}$ and $\mathrm{CDKi}$, clinical trials focused on combination therapy strategies as the first-line setting for treating MBC patients [25], [26].

Phase I/II study of PALOMA 1 trial assed the safety and tolerability of palbociclib plus letrozole as first-line treatment of postmenopausal HR+/HER2- MBC patients [25], [27]. As in previously preclinical studies, these phase II trial showed patients treated with palbociclib plus letrozole had a higher clinical benefit rate and PFS [17], [25], [28]. Besides, the cohort 2 of the clinical trial was therefore selected based on having CCND1 amplification and/or loss of $p 16$ in the primary tumour to seek a possible biomarker of efficacy, without it could be shown [28] .

Palbociclib plus letrozole was approved as first-line treatment in postmenopausal Luminal MBC patients with no prior treatment for the advanced disease after confirmed palbociclib safety and efficacy in Phase III trial (PALOMA 2) [9], [25]. Later on, PALOMA 3 (Phase III) trial assessed the combination therapy palbociclib plus fulvestrant to treat advanced BC patients that had progressed on previous hormone therapies regardless of menopausal status. HR+/HER2- MBC patients treated with the combinatorial therapy had a higher clinical benefit rate and PFS [12], [17], [29].

In the MONALEESA-2 (Phase III) trial, ribociclib plus letrozole was assessed as first-line therapy in postmenopausal patients with HR+/HER2- MBC. Patients significantly improved PFS and the toxicities were manageable [30], [31]. 
Next, the combination of ribociclib plus fulvestrant was assessed in MONALEESA-3 (Phase III) trial. It included postmenopausal patients who progress to (neo) adjunvant ET or presented de novo MBC, who were either treatment naive or received $\leq 1$ line of prior ET in the advanced disease setting. The results reported significant PFS improvements irrespective of prior ET [8], [30]. In MONALEESA-7 (Phase III) trial was approved the combination of ribociclib plus goserelin and tamoxifen or aromatase inhibitors (AI) in pre-menopausal MBC patients. It was reported PFS and overall survival (OS) was higher in the combinatorial therapy than in the placebo group [30], [32].

The endpoint of MONARCH 1 (Phase II) trial was determine the activity of abemaciclib as single-agent on continuous schedule in HR+/ HER2- MBC patients who progress on ET and/or chemotherapy [33]. The antitumour activity and manageable toxicities encourage the development of numerous trials to investigate abemaciclib in combination with ET in the first- and second-line settings. In MONARCH 2 (Phase III) trial the combination of abemaciclib plus fulvestrant was approved in MBC patients who progressed during (neo)-adjuvant or first-line ET, regardless of menopausal status [34]. The polytherapy improved objective response rate, OS and PFS, mainly in patients with poor prognosis factors such as visceral metastasis and endocrine resistance [34], [35]. Later, the combination of abemaciclib plus a non-steroidal AI was assessed in MONARCH 3 (Phase III) trial. The polytherapy significantly improved PFS and objective response rate with a tolerable safety profile as initial treatment for HR+/HER2- advanced BC [36].

Preclinical cell line studies have revealed some candidate resistance mechanisms such as upregulation of $\mathrm{Pi} 3 \mathrm{~K} / \mathrm{AKT} / \mathrm{mTOR}$ pathway, loss of RB1, acquired mutations in RB1 inhibitors, FGFR amplification or mutation, upregulation of PDK1, MYC or SKYPE and overexpression of CDK4/6. Likewise, the formation of CCNE-CDK2 and CCND1-CDK2 complexes can control cell cycle progression after CCND1-CDK4/6 inhibition. Wander et al. identified 8 possible resistance mechanism in patients resistant to CDKi: $R B 1$ allelic disruption, amplification and/or mutation in $A K T, R A S, A U R K A, C C N E 2, F G F R 2$ and ERBB2 amplification and loss of ESR1. Later on, these alterations were confirmed in resistant cell lines to CDK inhibitors. Then, preclinical evidence suggests that different cell cycle regulators and oncogenic drivers may be involved in CDKi resistance [37]. However, it remains to be seen whether these mechanisms identified in vitro are clinically relevant in drug-treated patients.

\section{Deciphering resistance mechanisms through Liquid biopsy analysis}

The current molecular cancer studies are based on primary tumour biopsies. Despite its extended use, this technique has multiple downsides: invasiveness, no representation of tumour genetic landscape and its inability to perform serial testing [38]. Tumours are heterogeneous and dynamic units that evolve throughout the disease, sometimes conditioned by the selective pressure exerted by the different treatments received. Therefore, primary biopsy data may not provide real information on current molecular characteristics and biopsies from metastases are iatrogenic and may not represent tumour heterogeneity [39]. Besides, in those patients who suffer from metastasis, sometimes tissue biopsies are not always feasible due to inaccessible tumour sites or the impossibility to sample multiple metastatic sites. In the last decade, it has been technically developed liquid biopsy (LB) as a new diagnostic approach to overcome tissue biopsy limitations. It is based on sampling biological fluids from patients (blood, urine, saliva, etc) to analyse tumour material as circulating tumour cells (CTCs), circulating tumour DNA (ctDNA) and tumour-derived extracellular vesicles (EVs) (Figure 2). Additionally, circulating tumour-derived proteins, circulating tumour RNA and tumour-bearing platelets are other components of LB with diagnostic or prognostic potential [40]. These tumour entities allow assessing the heterogeneity of the tumour, track its genomic evolution during treatment and give more information about the biology behind the metastatic development [41], [42]. Therefore, LB is useful to monitor therapy 
response and detect resistance which in turn, can help oncologist to predict the progression of the disease, the failure of treatment [43] and the selection of personalized therapies.

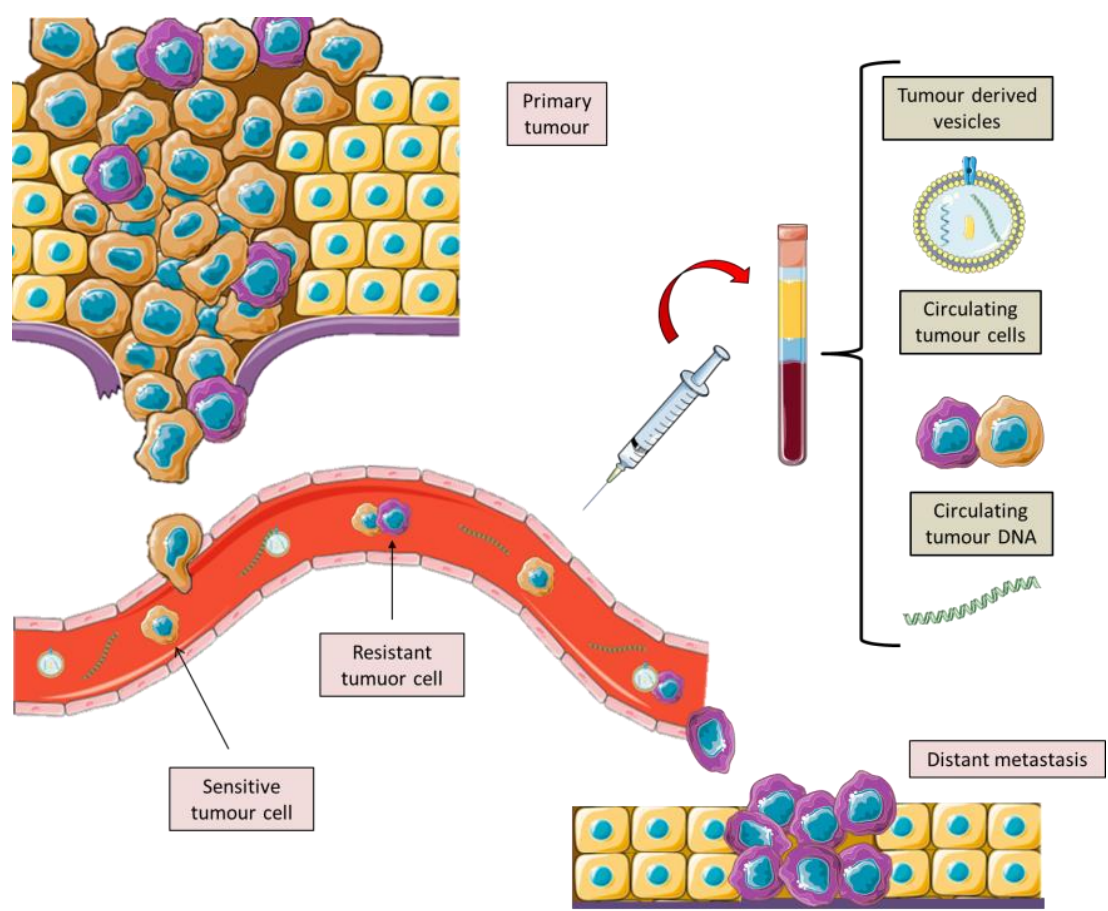

Figure 2. Scheme of metastatic progression and potential for liquid biopsy. In the blood stream there are different subpopulations of CTCs. Those that have bypassed the therapy and have become resistant are responsible for the recurrence and progression of the disease to distal locations. Besides, liquid biopsy permits the sampling of other tumour entities such as ctDNA or extracellular vesicles. The ctDNA represents the mutational status of the tumour. A serial liquid biopsy throughout therapy is very useful for studying the appearance of resistance to treatments.

\section{Circulating tumour DNA (ctDNA) analysis as a promising tumour biomarker}

The ctDNA analysis is an attractive minimally invasive opportunity for genomic study and searches for biomarkers in cancer patients. The amount of ctDNA depends not solely on the amount of death cells but also in the metabolism of the tumour, tumour location, vascularization, rate of proliferation, etc. It is released in the blood stream both passively and actively from primary tumours, CTCs and metastasis [44-46]. The ctDNA analysis can be used for diagnosis, to select targeted therapy, for detection of residual tumours and metastases, but also to detect clinical progression identifying resistance mutations [45], [46]. Indeed, in NSCLC (Non-small-cell lung carcinoma) patients, ctDNA has been approved for target therapy selection in advance stages and also in early stages (50\%) [47].

Currently, a great variety of studies are assessing the clinical utility of ctDNA in HR+/HER2- MBC treated with CDKi plus ET (Table 1). Despite no association between biomarkers and therapy response was detected in primary tumours [25], [28], [29], some alterations in ctDNA were identified. Subclonal mutations in RB1 at end of treatment were detected in $5 \%$ of patients treated with palbociclib or ribociclib plus ET. The clinical prevalence of RB1 mutations in primary BC tumours is rare, but in patients resistant to CDK4/6 inhibitors with prior endocrine therapy is unknown. These alterations were the result of selective therapy pressure, since were not detected in the ctDNA obtained before exposure to palbociclib or ribociclib. Also, as these mutations were part of a subclonal population, are difficult to detect in tumour derived material [4], [48]. Furthermore, RB1 mutations were only selected in tumours wild-type for 
ESR1 mutations, which could suggest that RB1 mutations could be selected when fulvestrant efficacy is not compromised by ESR1 mutation, proposing divergent routes to resistance [48].

Some studies observed ESR1 subclonal mutations in the ligand-binding domain in patients resistant to polytherapy, while others found endocrine-resistant patients are sensitive to CDKi regardless of ESR1 status [49]-[51]. A reduction in ctDNA abundance was also observed after two weeks of therapy, but did not improve PFS and did not predict sensitivity [52]. Besides, it was observed patients with ESR1 mutations at baseline had a worse PFS than those with wild type mutations, probably due to differentially mutation sensitivity to therapy. Then, it was noticed that ESR1 early clonal dynamics could predict clonal composition at relapse. Loss of ESR1 mutation at end of treatment was more frequent in patients on palbociclib and fulvestrant than those on fulvestrant and placebo. Another study found substantial ESR1 loss and gain through treatment reflects individual tumour subclones as well as the loss of sensible subclones due to therapy pressure [4]. Further analyses are required to comprehend the role of ESR1 mutations in polytherapy resistance. Regarding PI3K alterations, it was described as a gene with a strong pattern of variant acquisition and loss of relatively few clones on treatment [4], [29]. There was no-association between PI3K alterations and PFS, the benefit of the combinatorial therapy or HR status [29]. However, it was observed that a reduction in PIK3CA ctDNA level after two weeks of treatment predicted long term clinical outcome (4 months vs 11 months) [52].

Table 1. Summary of studies in luminal patients treated with CDKi by liquid biopsy . 


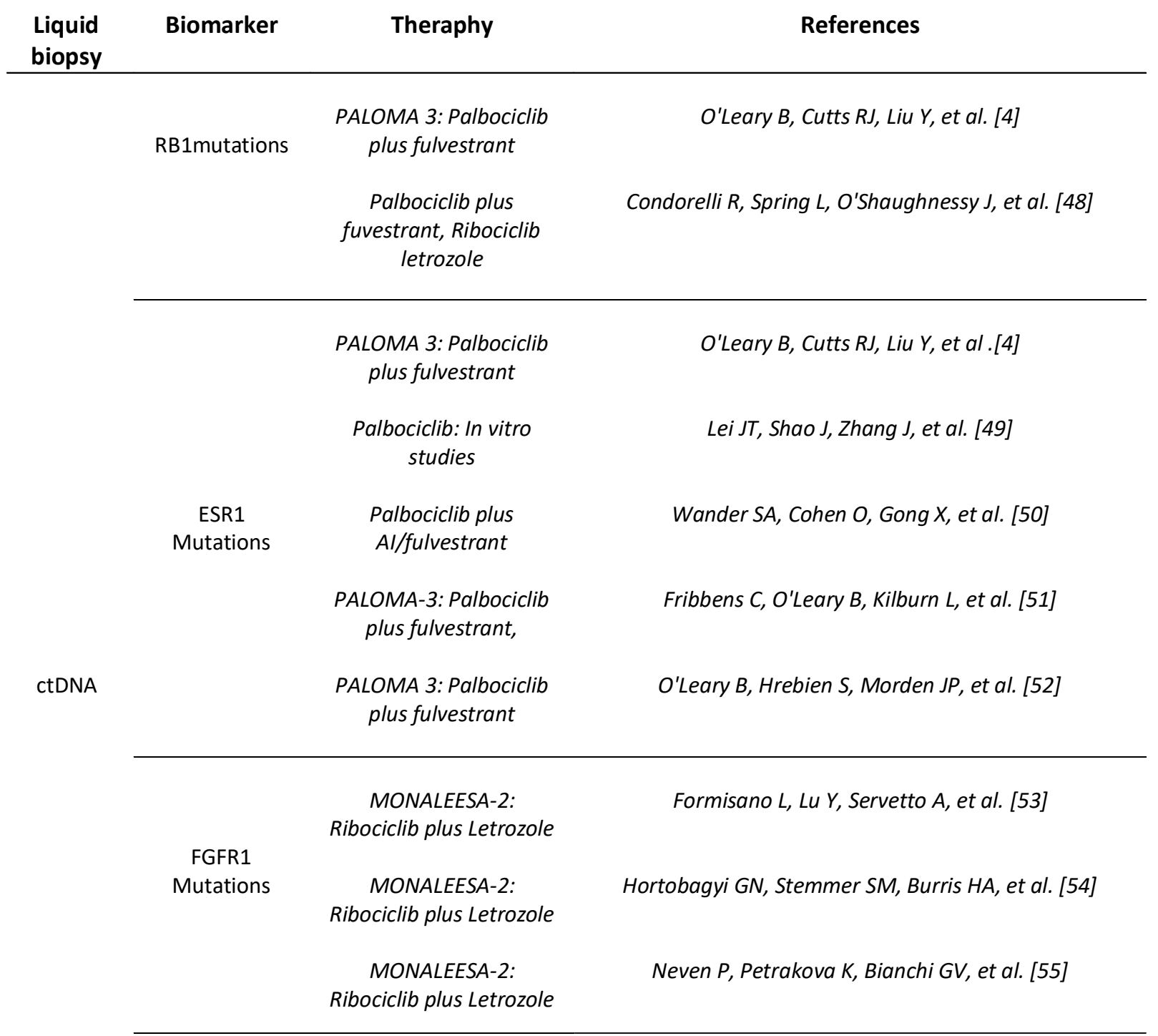

\section{PALOMA 3: Palbociclib O'Leary B, Cutts RJ, Liu Y, et al. [4]}

PI3K Mutations plus fulvestrant

PALOMA 3: Palbociclib Cristofanilli M, Turner NC, Bondarenko l, et al. [29] plus fulvestrant

PALOMA 3: Palbociclib O'Leary B, Hrebien S, Morden JP, et al. [52] plus fulvestrant

$\begin{array}{ccc}\text { mRNA CDK9/4 } & \text { CDKi plus endoncrine } \\ \text { and TK1 } & \text { theraphy } & \text { Del Re M, Bertolini I, Crucitta S, et al. [63] }\end{array}$

Exosomes

miRNA 432-5-p

Palbociclib plus letrozole/fulvestrant

Cornell L, Wander SA, Visal T, Wagle N, Shapiro GI [64]

Another study conducted by Formisano et al. detected FGFR1 overexpression decreased PFS. Besides, they identified mRNA overexpression and gene amplification of FGFR1 as a possible mechanism to reduce sensitivity to palbociclib and fulvestrant treatment by performing in vitro experiments [53]. After analysing ctDNA from HR+/HER- MBC patients that progressed after palbociclib and fulvestrant treatment, they found that up to $41 \%$ of the patients had an 
FGFR1 alteration, suggesting a possible connection between FGFR1 mutations and progression. It was also analysed the ctDNA to test the efficacy of the combination of ribociclib plus letrozole. Although only $5 \%$ of the patients had an alteration in FGFR1, these mutations correlated with worse patient's outcome. Besides, the analysis of FGFR1 mRNA expression in tumour samples shows that patients with high FGFR1 mRNA expression had worse PFS compared to those who had low FGFR1 mRNA expression when treated with letrozole plus ribociclib [53]. In other studies, molecular alterations in ctDNA were analysed to identify biomarkers that predict ribociclib plus letrozole therapy response. This polytherapy improved PFS regardless of ctDNA genetic alterations at baseline: PIK3CA, TP53, CDH1, FGFR1, cell cycle-related genes or genes involved in receptor tyrosine kinase signalling [54]. However, Neven et al. found shorter PFS was correlated with altered gene status irrespective of treatment (polytherapy or letrozole alone) [54], [55].

In conclusion, it was observed acquisition of mutations at end of treatment was related with longer PFS in patients who progress later on the polytherapy. It is likely tumours that progress early does not acquire mutations due to lack of treatment pressure. Then, other mechanisms of resistance may dominate in early progression, so it is important to consider intrinsic resistance to select the next line of treatment. In addition, it was described fulvestrant as a major genetic driver of resistance to combinatorial therapy. One possible explanation is tumours are able to adapt to CDK4/6 inhibitors if ESR1 signalling is not correctly suppressed [4].

Currently, an active randomized phase III trial (PADA-1) aims to evaluate the efficacy of switching hormone therapy (from AI to fulvestrant) combined with palbociclib, assessing ESR1 mutations in ctDNA. Likewise, it will determine the safety of hormone therapy and palbociclib. Thus, ESR1 mutations (E380, L536, Y537 and D538 hotspots) will be monitored at baseline and after each cycle of treatment by ddPCR [56]. As preliminary results, ESR1 mutations were uncommon in patients no-treated with $\mathrm{AI}$ in the neoadjuvant setting. Besides, one-month treatment with palbociclib and AI decreases ESR1 mutation rate [57], [58].

\section{Circulating Tumour Cells, a possible biomarker to manage HR+/HER2- MBC patients}

Cancer heterogeneity results in tumour cells subpopulations that have different rates of proliferation, aggressiveness and drug sensitivity. These cancer tumour cells are released into the blood circulation actively by epithelial-mesenchymal transition or passively detached from primary tumour or metastasis as single cells or clusters, which have a higher metastatic potential. Thus, the presence in blood of $\geq 5$ Circulating Tumour cells (CTCs) per 7.5 $\mathrm{mL}$ were associated with poor outcome in metastatic breast and prostate cancer, while $\geq 3$ CTCs per $7.5 \mathrm{~mL}$ in colorectal cancer patients [44]. CellSearch ${ }^{\circledR}$ system (Menarini Silicon Biosystems, Inc) is the only platform validated by FDA for CTC detection and enumeration. It is an immunomagnetic enrichment method that used epithelial antibodies, EpCAM and cytokeratins 8, 18 and/or 19, to positively enrich CTCs. Nevertheless, it ignores CTC subpopulations with mesenchymal or stemness phenotype [44]. Despite the technological advances, the low number of CTCs in the blood system is still a hindrance to their characterization [59]. Thus, sampling higher volumes of blood by leukapheresis is an alternative that is recently being explored. Also, studies at the single-cell level are shedding light on tumourigenesis therapy response or even mechanism of resistance in BC and others tumour types [44], [60]. The single CTC analysis unravels the heterogeneity of the tumour and makes it possible to study resistant clones derived from the selective pressure of multiple therapies received throughout the disease. Thus, a study by De Luca et al. carried out in a patient with breast cancer, observed that most of the CTC mutations detected at the beginning of the study disappeared during treatment and new mutations emerged [61]. 
The study of CTCs will allow omics analysis (gene expression, proteins, metabolites, etc.) as well as functional tests in vitro and in vivo. To fully understand the metastatic process and the underlying resistance mechanisms, it is essential to incorporate the analysis of CTCs in the metastatic clinical setting. Regarding HR+/HER2- metastatic BC patients treated with the combinatorial therapy CDKi and ET, it has not been reported any study based on CTCs account or molecular analysis in a representative cohort. CTCs gene expression analysis before and after therapy could help to decipher potential resistance or response mechanisms in these patients (González-Conde et al., unpublished data). Interestingly, in 2020 Koch C et al. established an ER+ breast CTC line derived from a patient with Luminal metastatic breast cancer. It was demonstrated the cell line is genetically identical to the original CTCs, something never described before. Hence, having CTC-cell lines is a good model to test drug activity and to decipher the intrinsic mechanisms involved in the metastatic cascade. It has already observed palbociclib reduced cell cycle growth even at low doses in this novel CTC cell line [59].

\section{Extracellular vesicles and resistance mechanisms to CDKi}

Exosomes are extracellular vesicles with a diameter between 50-100 nm that can be released from many cell types. Among its functions, exosomes have been linked to important roles in cancer biology such as tumorigenesis, angiogenesis, invasion and metastasis. It is also described in the literature that exosomes can transmit drug resistance through functional proteins and microRNAs (miRNA) [62].

An interesting work performed by Del Re et al. identified some promising biomarkers to monitor treatment outcome in HR+/HER2- MBC patients treated with CDKi and endocrine therapy. In this study, the exosomes derived from the plasma of 40 patients were analysed. The patients with elevated levels of CDK4 mRNA in their exosomes responded to the treatment and had prolonged PFS. Besides, an increase in CDK9 and TK1 mRNA copies in the exosomes extracted from the plasma was related to clinical resistance. Despite the lack of more studies, these preliminary results set exosomes as a promising biomarker to monitor the CDKi treatment outcome [63].

In fact, in 2019 Cornell and colleagues demonstrated a new exosome-mediated mechanism of acquired resistance to CDKi as palbociclib. This mechanism is independent of genetic mutations and is acquired through extracellular signalling, involving exosomal miRNA [64]. They observed an increase in protein and mRNA expression of CDK6 in palbociclib resistant BC cell lines. The CDK6 knockdown re-sensitized the cells to palbociclib treatment, indicating this cyclin as a key mediator of the resistance mechanism. Through experiments of co-culture, they found that palbociclib sensible cell lines could acquire resistance to the drug when co-cultured with resistant cells or with their exosomes, suggesting that the resistance could be transmitted through extracellular vesicles. The analysis of the exosomes in these cell lines identified the miRNA 432-5-p as a possible mediator of CDKi resistance [64]. Next, they found that miR-432-5p interacts predominantly with numerous genes from the TGF- $\beta$ pathway. The expression of miR-432-5p was assessed on tumour biopsies from patients with ER+ MBC. The expression of miR-432-5p was found to be 1,8 fold higher in biopsies from ER+ BC patients with intrinsic or acquired CDKi resistance when compared to patients with sensitive tumour to the treatment. It was also observed a 2,7 fold decrease in SMAD4 expression in resistant tumours, indicating a TGF- $\beta$ pathway suppression mediated by miR-432-5p. Using both in vitro and in vivo models, the authors provided evidence of the loss of acquired resistance through drug removal suggesting possible reuse of CDKi after adequate drug breaks [64]. Although all the analysis were performed on primary tumours, this work supports that the study of exosomes could be useful to monitor the response to treatment and the related resistance mechanisms. 


\section{Conclusions}

The liquid biopsy is being a fundamental tool to study tumour heterogeneity, the main cause of therapeutic failure in cancer patients. Liquid biopsy provides an insight into the dynamic molecular profile of the primary tumour and its metastasis in a non-invasive and real-time approach [54]. A great variety of trials have demonstrated the benefits of combined CDK4/6 inhibitors plus endocrine therapy in HR+/HER2- MBC, such as increasing PFS regardless of menopausal status, prior therapies, endocrine sensitivity and type of metastasis [24], [57]. However, certain limitations were not resolved such as lack of predictive biomarkers to select patients or to detect resistance [25] and is one of the current topics in the context of luminal metastatic breast cancer.

In this regard, studies carried out in ctDNA point to the appearance of subclonal mutations in ESR1 or at the end of polytherapy. Besides, some patients with endocrine resistance are sensitive to CDKi regardless of ESR1 status. This suggests that fulvestrant could be a resistance driver, but expanded clinical studies are needed in this regard. Other proposed biomarkers as FGFR or PI3K lead to contradictory data that do not allow to reach reliable conclusions.

Despite the enormous potential of CTCs, there are no reported works of CTCs gene expression in these patients. Deciphering changes in expression after combined therapy, especially in resistant CTCs, may be a milestone that allows interpreting the underlying resistance mechanisms. This would be of special interest since those CTCs that bypass therapy, can colonize distal organs and contribute to the progression of the disease. In this sense, we have preliminary data that suggest that the analysis of CTCs can contribute in a relevant way. Concerning exosomes, some publications point to them as very promising biomarkers in the field, but it is necessary to continue with these investigations.

One limitation of studing patients treated with a combined therapy is the lack of knowledge about the contribution of each treatment or if the resistance is due to the action of both drugs. Furthermore, in the clinical context, it remains to be defined whether the mutational state prior therapy determine therapy efficacy . Besides, it should be noted that in tumours that progress early, driver gene mutations evolution is infrequent, probably due to the lack of selective pressure. Therefore, these patients are of special interest due to intrinsic resistance.

Owing to the genetic complexity of cancer and possible mechanisms of acquired resistance, simple models of genetically encoded sensitivity do not reflect the patients' genetic landscape [6]. Hence, is not seeking a standard treatment sequence, but to know the profile of each patient at a certain time to adapt the most beneficial therapy, which means precision medicine.

For all this, the future outlook should be based on molecular characterisation of the primary tumour, metastasis as well as tumour-derived material (ctDNA, CTC or EVs) at different time points in the metastatic clinical setting. Comprehensive Liquid biopsy analysis of tumour material will change the current clinical paradigm of luminal BC patients completely. Several ongoing clinical trials consider the study of ctDNA but do not include other circulating tumoural entities yet. However, the clinical implementation of liquid biopsy is underway and despite current technological limitations, it is a matter of time before it uses is universal [25], [44], [46]. 
Author Contributions: CC was involved in the design of the manuscript. MGC, CYG and CC wrote the original draft. All the authors reviewed and edited the final version.

Funding: This research was funded by Roche-Chus Joint Unit (IN853B 2018/03), funded by Axencia Galega de Innovación (GAIN), Vicepresidencia Segunda e Consellería de Economía, Empresa e Innovación, and by Instituto de Salud Carlos III (ISCiii), grants PI18/00183 and FI19/00140 (MGC support), co-financed by the European Regional Development Fund (FEDER). CYG is supported by funds from "Programa de ayudas a la etapa predoctoral» from Xunta de Galicia (Consellería de Educación, Universidad y Formación Profesional).

Conflicts of Interest: R.L.-L. reports grants and personal fees from Roche, Merck, AstraZeneca, Bayer, Pharmamar, Leo, and personal fees and non-financial support from Bristol-Myers Squibb and Novartis, outside of the submitted work. The other authors declare no conflict of interest.

\section{References}

1. F. Bray, J. Ferlay, I. Soerjomataram, R. L. Siegel, L. A. Torre, y A. Jemal, «Global cancer statistics 2018: GLOBOCAN estimates of incidence and mortality worldwide for 36 cancers in 185 countries», CA Cancer J Clin, vol. 68, n.o 6, pp. 394-424, nov. 2018, doi: 10.3322/caac. 21492 .

2. T. Sorlie et al., «Repeated observation of breast tumor subtypes in independent gene expression data sets», Proc Natl Acad Sci U S A, vol. 100, n.o 14, pp. 8418-8423, jul. 2003, doi: 10.1073/pnas.0932692100.

3. A. Rani, J. Stebbing, G. Giamas, y J. Murphy, «Endocrine Resistance in Hormone Receptor Positive Breast Cancer-From Mechanism to Therapy», Front. Endocrinol., vol. 10, 2019, doi: 10.3389/fendo.2019.00245.

4. B. O'Leary et al., «The Genetic Landscape and Clonal Evolution of Breast Cancer Resistance to Palbociclib plus Fulvestrant in the PALOMA-3 Trial», Cancer Discov, vol. 8, n.o 11, pp. 1390-1403, nov. 2018, doi: 10.1158/2159-8290.CD-18-0264.

5. E. A. Musgrove y R. L. Sutherland, «Biological determinants of endocrine resistance in breast cancer», Nat Rev Cancer, vol. 9 , n.o 9, pp. 631-643, sep. 2009, doi: 10.1038/nrc2713.

6. E. S. Knudsen y A. K. Witkiewicz, «The Strange Case of CDK4/6 Inhibitors: Mechanisms, Resistance, and Combination Strategies», Trends Cancer, vol. 3, n.o 1, pp. 39-55, ene. 2017, doi: 10.1016/j.trecan.2016.11.006.

7. D. J. Slamon et al., «Overall Survival with Ribociclib plus Fulvestrant in Advanced Breast Cancer», N Engl J Med, vol. 382 , n.o 6, pp. 514-524, feb. 2020, doi: 10.1056/NEJMoa1911149.

8. D. J. Slamon et al., «Phase III Randomized Study of Ribociclib and Fulvestrant in Hormone Receptor-Positive, Human Epidermal Growth Factor Receptor 2-Negative Advanced Breast Cancer: MONALEESA-3», J Clin Oncol, vol. 36, n.o 24, pp. 2465-2472, ago. 2018, doi: 10.1200/JCO.2018.78.9909.

9. R. S. Finn et al., «Palbociclib and Letrozole in Advanced Breast Cancer», N Engl J Med, vol. 375, n.o 20, pp. 1925-1936, nov. 2016, doi: 10.1056/NEJMoa1607303.

10. D. Tripathy et al., «Ribociclib plus endocrine therapy for premenopausal women with hormone-receptor-positive, advanced breast cancer (MONALEESA-7): a randomised phase 3 trial», Lancet Oncol, vol. 19, n.o 7, pp. 904-915, jul. 2018, doi: 10.1016/S1470-2045(18)30292-4.

11. G. W. Sledge et al., «The Effect of Abemaciclib Plus Fulvestrant on Overall Survival in Hormone Receptor-Positive, ERBB2-Negative Breast Cancer That Progressed on Endocrine Therapy-MONARCH 2: A Randomized Clinical Trial», JAMA Oncol, sep. 2019, doi: 10.1001/jamaoncol.2019.4782.

12. N. C. Turner et al., «Overall Survival with Palbociclib and Fulvestrant in Advanced Breast Cancer», N Engl J Med, vol. 379 , n.o 20, pp. 1926-1936, nov. 2018, doi: 10.1056/NEJMoa1810527.

13. M. P. Goetz et al., «MONARCH 3: Abemaciclib As Initial Therapy for Advanced Breast Cancer», J Clin Oncol, vol. 35, n.o 32, pp. 3638-3646, nov. 2017, doi: 10.1200/JCO.2017.75.6155.

14. P. Razavi et al., «The Genomic Landscape of Endocrine-Resistant Advanced Breast Cancers», Cancer Cell, vol. 34, n.o 3, pp. 427-438.e6, sep. 2018, doi: 10.1016/j.ccell.2018.08.008.

15. L. M. Spring, S. A. Wander, F. Andre, B. Moy, N. C. Turner, y A. Bardia, «Cyclin-dependent kinase 4 and 6 inhibitors for hormone receptor-positive breast cancer: past, present, and future», Lancet, vol. 395, n.o 10226, pp. 817-827, mar. 2020, doi: 10.1016/S0140-6736(20)30165-3.

16. A. Prat et al., "Clinical implications of the intrinsic molecular subtypes of breast cancer», Breast, 2015, doi: 10.1016/j.breast.2015.07.008.

17. N. Portman, S. Alexandrou, E. Carson, S. Wang, E. Lim, y C. E. Caldon, «Overcoming CDK4/6 inhibitor resistance in ER-positive breast cancer», Endocr Relat Cancer, vol. 26, n.o 1, pp. R15-R30, ene. 2019, doi: 10.1530/ERC-18-0317.

18. M. A. Dickson, «Molecular pathways: CDK4 inhibitors for cancer therapy», Clin Cancer Res, vol. 20, n.o 13, pp. 3379-3383, jul. 2014, doi: 10.1158/1078-0432.CCR-13-1551.

19. K. Pandey et al., «Molecular mechanisms of resistance to CDK4/6 inhibitors in breast cancer: A review», Int J Cancer, vol. 145, n.o 5, pp. 1179-1188, sep. 2019, doi: 10.1002/ijc.32020.

20. A. McCartney et al., «Mechanisms of Resistance to CDK4/6 Inhibitors: Potential Implications and Biomarkers for Clinical Practice», Front. Oncol., vol. 9, 2019, doi: 10.3389/fonc.2019.00666. 
21. U. Asghar, A. K. Witkiewicz, N. C. Turner, y E. S. Knudsen, «The history and future of targeting cyclin-dependent kinases in cancer therapy», Nat Rev Drug Discov, vol. 14, n.o 2, pp. 130-146, feb. 2015, doi: 10.1038/nrd4504.

22. S. Tadesse et al., «Discovery and pharmacological characterization of a novel series of highly selective inhibitors of cyclin-dependent kinases 4 and 6 as anticancer agents», Br J Pharmacol, vol. 175, n.o 12, pp. 2399-2413, jun. 2018, doi: 10.1111/bph.13974.

23. S. Kalra, G. Joshi, A. Munshi, y R. Kumar, «Structural insights of cyclin dependent kinases: Implications in design of selective inhibitors», Eur J Med Chem, vol. 142, pp. 424-458, dic. 2017, doi: 10.1016/j.ejmech.2017.08.071.

24. V. Rossi et al., «Should All Patients With HR-Positive HER2-Negative Metastatic Breast Cancer Receive CDK 4/6 Inhibitor As First-Line Based Therapy? A Network Meta-Analysis of Data from the PALOMA 2, MONALEESA 2, MONALEESA 7, MONARCH 3, FALCON, SWOG and FACT Trials», Cancers (Basel), vol. 11, n.o 11, oct. 2019, doi: 10.3390/cancers11111661.

25. A. De Luca et al., «Pharmacokinetic drug evaluation of palbociclib for the treatment of breast cancer», Expert Opin Drug Metab Toxicol, vol. 14, n.o 9, pp. 891-900, sep. 2018, doi: 10.1080/17425255.2018.1514720.

26. A. DeMichele et al., «CDK 4/6 inhibitor palbociclib (PD0332991) in Rb+ advanced breast cancer: phase II activity, safety, and predictive biomarker assessment», Clin Cancer Res, vol. 21, n.o 5, pp. 995-1001, mar. 2015, doi: 10.1158/1078-0432.CCR-14-2258.

27. «Study Of Letrozole With Or Without Palbociclib (PD-0332991) For The First-Line Treatment Of Hormone-Receptor Positive Advanced Breast Cancer - Tabular View - ClinicalTrials.gov». https://clinicaltrials.gov/ct2/show/record/NCT00721409 (accedido ene. 27, 2021).

28. R. S. Finn et al., «The cyclin-dependent kinase $4 / 6$ inhibitor palbociclib in combination with letrozole versus letrozole alone as first-line treatment of oestrogen receptor-positive, HER2-negative, advanced breast cancer (PALOMA-1/TRIO-18): a randomised phase 2 study», Lancet Oncol, vol. 16, n.o 1, pp. 25-35, ene. 2015, doi: 10.1016/S1470-2045(14)71159-3.

29. M. Cristofanilli et al., «Fulvestrant plus palbociclib versus fulvestrant plus placebo for treatment of hormone-receptor-positive, HER2-negative metastatic breast cancer that progressed on previous endocrine therapy (PALOMA-3): final analysis of the multicentre, double-blind, phase 3 randomised controlled trial», Lancet Oncol, vol. 17, n.o 4, pp. 425-439, abr. 2016, doi: 10.1016/S1470-2045(15)00613-0.

30. D. A. Yardley, «MONALEESA clinical program: a review of ribociclib use in different clinical settings», Future Oncol, vol. 15, n.o 23, pp. 2673-2686, ago. 2019, doi: 10.2217/fon-2019-0130.

31. G. N. Hortobagyi et al., «Updated results from MONALEESA-2, a phase III trial of first-line ribociclib plus letrozole versus placebo plus letrozole in hormone receptor-positive, HER2-negative advanced breast cancer», Ann Oncol, vol. 29, n.o 7, pp. 1541-1547, jul. 2018, doi: 10.1093/annonc/mdy155.

32. S. A. Hurvitz et al., «Phase III MONALEESA-7 trial of premenopausal patients with HR+/HER2- advanced breast cancer $(\mathrm{ABC})$ treated with endocrine therapy \pm ribociclib: Overall survival (OS) results.», JCO, vol. 37, n.o 18_suppl, pp. LBA1008-LBA1008, jun. 2019, doi: 10.1200/JCO.2019.37.18_suppl.LBA1008.

33. M. N. Dickler et al., «MONARCH 1, A Phase II Study of Abemaciclib, a CDK4 and CDK6 Inhibitor, as a Single Agent, in Patients with Refractory HR+/HER2- Metastatic Breast Cancer», Clin Cancer Res, vol. 23, n.o 17, pp. 5218-5224, sep. 2017, doi: 10.1158/1078-0432.CCR-17-0754.

34. P. A. Kaufman et al., «Health-Related Quality of Life in MONARCH 2: Abemaciclib plus Fulvestrant in Hormone Receptor-Positive, HER2-Negative Advanced Breast Cancer After Endocrine Therapy», Oncologist, vol. 25, n.o 2, pp. e243-e251, feb. 2020, doi: 10.1634/theoncologist.2019-0551.

35. G. W. Sledge et al., «MONARCH 2: Abemaciclib in Combination With Fulvestrant in Women With HR+/HER2- Advanced Breast Cancer Who Had Progressed While Receiving Endocrine Therapy», JCO, vol. 35, n.o 25, pp. 2875-2884, jun. 2017, doi: 10.1200/JCO.2017.73.7585.

36. S. Johnston et al., «MONARCH 3 final PFS: a randomized study of abemaciclib as initial therapy for advanced breast cancer», npj Breast Cancer, vol. 5, n.o 1, Art. n.o 1, ene. 2019, doi: 10.1038/s41523-018-0097-z.

37. K. L. Thu, I. Soria-Bretones, T. W. Mak, y D. W. Cescon, «Targeting the cell cycle in breast cancer: towards the next phase», Cell Cycle, vol. 17, n.o 15, pp. 1871-1885, 2018, doi: 10.1080/15384101.2018.1502567.

38. D. Fernández-Lázaro, J. L. García Hernández, A. Caballero García, A. Caballero del Castillo, M. Villaverde Hueso, y J. J. Cruz-Hernández, «Clinical Perspective and Translational Oncology of Liquid Biopsy», Diagnostics, vol. 10, n.o 7, p. 443, jun. 2020, doi: 10.3390/diagnostics10070443.

39. M. W. Fittall y P. Van Loo, «Translating insights into tumor evolution to clinical practice: promises and challenges», Genome Med, vol. 11, n.o 1, p. 20, mar. 2019, doi: 10.1186/s13073-019-0632-z.

40. J. Wu et al., «Tumor circulome in the liquid biopsies for cancer diagnosis and prognosis», Theranostics, vol. 10, n.o 10, pp. 4544-4556, mar. 2020, doi: 10.7150/thno.40532.

41. C. Alix-Panabières y K. Pantel, «Clinical Applications of Circulating Tumor Cells and Circulating Tumor DNA as Liquid Biopsy», Cancer Discov, vol. 6, n.o 5, pp. 479-491, may 2016, doi: 10.1158/2159-8290.CD-15-1483.

42. S. Junqueira-Neto, I. A. Batista, J. L. Costa, y S. A. Melo, «Liquid Biopsy beyond Circulating Tumor Cells and Cell-Free DNA», Acta Cytologica, p. 10.

43. J. Wang, S. Chang, G. Li, y Y. Sun, «Application of liquid biopsy in precision medicine: opportunities and challenges», Front. Med., vol. 11, n.o 4, pp. 522-527, dic. 2017, doi: 10.1007/s11684-017-0526-7.

44. P. Fici, «Cell-Free DNA in the Liquid Biopsy Context: Role and Differences Between ctDNA and CTC Marker in Cancer Management», Methods Mol Biol, vol. 1909, pp. 47-73, 2019, doi: 10.1007/978-1-4939-8973-7_4.

45. L. Keller, Y. Belloum, H. Wikman, y K. Pantel, «Clinical relevance of blood-based ctDNA analysis: mutation detection and beyond», Br J Cancer, vol. 124, n.o 2, pp. 345-358, ene. 2021, doi: 10.1038/s41416-020-01047-5. 
46. N. O. Tuaeva et al., «Translational Application of Circulating DNA in Oncology: Review of the Last Decades Achievements», Cells, vol. 8, n.o 10, oct. 2019, doi: 10.3390/cells8101251.

47. E. Rijavec, S. Coco, C. Genova, G. Rossi, L. Longo, y F. Grossi, «Liquid Biopsy in Non-Small Cell Lung Cancer: Highlights and Challenges», Cancers (Basel), vol. 12, n.o 1, dic. 2019, doi: 10.3390/cancers12010017.

48. R. Condorelli et al., «Polyclonal RB1 mutations and acquired resistance to CDK 4/6 inhibitors in patients with metastatic breast cancer», Ann Oncol, vol. 29, n.o 3, pp. 640-645, mar. 2018, doi: 10.1093/annonc/mdx784.

49. J. T. Lei et al., «Functional Annotation of ESR1 Gene Fusions in Estrogen Receptor-Positive Breast Cancer», Cell Rep, vol. 24, n.o 6, pp. 1434-1444.e7, ago. 2018, doi: 10.1016/j.celrep.2018.07.009.

50. S. A. Wander et al., «The genomic landscape of intrinsic and acquired resistance to cyclin-dependent kinase $4 / 6$ inhibitors in patients with hormone receptor positive metastatic breast cancer», bioRxiv, p. 857839, nov. 2019, doi: 10.1101/857839.

51. C. Fribbens et al., «Plasma ESR1 Mutations and the Treatment of Estrogen Receptor-Positive Advanced Breast Cancer», J Clin Oncol, vol. 34, n.o 25, pp. 2961-2968, sep. 2016, doi: 10.1200/JCO.2016.67.3061.

52. B. O'Leary et al., «Early circulating tumor DNA dynamics and clonal selection with palbociclib and fulvestrant for breast cancer», Nat Commun, vol. 9, mar. 2018, doi: 10.1038/s41467-018-03215-x.

53. L. Formisano et al., «Aberrant FGFR signaling mediates resistance to CDK4/6 inhibitors in ER+ breast cancer», Nat Commun, vol. 10, n.o 1, p. 1373, mar. 2019, doi: 10.1038/s41467-019-09068-2.

54. G. N. Hortobagyi et al., «Abstract PD4-06: First-line ribociclib + letrozole in hormone receptor-positive, HER2-negative advanced breast cancer: Efficacy by baseline circulating tumor DNA alterations in MONALEESA-2», Cancer Res, vol. 78, n.o 4 Supplement, pp. PD4-PD4-06, feb. 2018, doi: 10.1158/1538-7445.SABCS17-PD4-06.

55. P. Neven et al., «Abstract PD2-05: Biomarker analysis by baseline circulating tumor DNA alterations in the MONALEESA-3 study», Cancer Res, vol. 79, n.o 4 Supplement, pp. PD2-PD2-05, feb. 2019, doi: 10.1158/1538-7445.SABCS18-PD2-05.

56. «PAlbociclib and Circulating Tumor DNA for ESR1 Mutation Detection - Tabular View - ClinicalTrials.gov». https://clinicaltrials.gov/ct2/show/record/NCT03079011 (accedido ene. 27, 2021).

57. O. Fiste, M. Liontos, K. Koutsoukos, E. Terpos, M. A. Dimopoulos, y F. Zagouri, «Circulating tumor DNA-based predictive biomarkers in breast cancer clinical trials: a narrative review», Ann Transl Med, vol. 8, n.o 23, p. 1603, dic. 2020, doi: 10.21037/atm-20-1175.

58. F.-C. Bidard et al., «Abstract PD2-06: Circulating ESR1 mutation detection rate and early decrease under first line aromatase inhibitor and palbociclib in the PADA-1 trial (UCBG-GINECO)», Cancer Res, vol. 79, n.o 4 Supplement, pp. PD2-PD2-06, feb. 2019, doi: 10.1158/1538-7445.SABCS18-PD2-06.

59. C. Koch et al., "Characterization of circulating breast cancer cells with tumorigenic and metastatic capacity», EMBO Mol Med, vol. 12, n.o 9, p. e11908, sep. 2020, doi: 10.15252/emmm.201911908.

60. N. V. Jordan et al., «HER2 expression identifies dynamic functional states within circulating breast cancer cells», Nature, vol. 537, n.o 7618, Art. n.o 7618, sep. 2016, doi: 10.1038/nature19328.

61. F. De Luca et al., «Mutational analysis of single circulating tumor cells by next generation sequencing in metastatic breast cancer», Oncotarget, vol. 7, n.o 18, pp. 26107-26119, may 2016, doi: 10.18632/oncotarget.8431.

62. W.-X. Chen et al., «Bioinformatics analysis of dysregulated microRNAs in exosomes from docetaxel-resistant and parental human breast cancer cells», CMAR, vol. Volume 11, pp. 5425-5435, jun. 2019, doi: 10.2147/CMAR.S201335.

63. M. Del Re et al., «Overexpression of TK1 and CDK9 in plasma-derived exosomes is associated with clinical resistance to CDK4/6 inhibitors in metastatic breast cancer patients», Breast Cancer Res Treat, vol. 178, n.o 1, pp. 57-62, nov. 2019, doi: 10.1007/s10549-019-05365-y.

L. Cornell, S. A. Wander, T. Visal, N. Wagle, y G. I. Shapiro, «MicroRNA-Mediated Suppression of the TGF- $\beta$ Pathway Confers Transmissible and Reversible CDK4/6 Inhibitor Resistance», Cell Rep, vol. 26, n.o 10, pp. 2667-2680.e7, mar. 2019, doi: 10.1016/j.celrep.2019.02.023. 